\title{
De Bolonia a España: constataciones y consideraciones sobre la reforma universitaria ${ }^{1}$
}

From Bologna to Spain: Findings and

Considerations about University Reform

\section{Gustavo Eduardo Toledo Lara*}

(iD) https://orcid.org/0000-0002-5104-9555

Tipo de Artículo: Informes de Investigación y ensayos inéditos

Doi: 10.17533/udea.unipluri.19.1.02

Cómo citar este artículo:

Toledo Lara, G. (2019). De Bolonia a España: constataciones y consideraciones sobre la reforma universitaria.

Uni-pluriversidad, 19(1), 14-32 doi: 10.17533/udea.unipluri.19.1.02

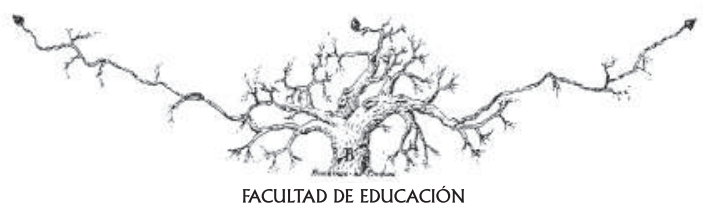

Recibido: 2018-12-09 • Aprobado: 2019-07-12

* Doctor por la Facultad de Educación de la Universidad de Salamanca, con estancia postdoctoral en la Universidad Autónoma de Madrid. Máster en Estudios Latinoamericanos, especialidad política, MSc. En Educación Superior (mención honorífica) y pedagogo. Profesor de la Facultad de Educación de la Universidad Internacional de La Rioja - España. 


\title{
Resumen
}

Se presenta un análisis sobre el Proceso de Bolonia visto desde la Universidad española. Las implicaciones reformistas de dicho proceso han impulsado un conjunto de modificaciones que se pueden revisar desde lo cualitativo con el objeto de determinar sus posibles efectos de cara a la educación universitaria de España. Por lo tanto, se asumió el método analítico interpretativo como línea metodológica para la realización de este ensayo, considerando la observación y análisis de fuentes primarias y secundarias. Además, la observación realizada a través de los diferentes gobiernos y de las normativas sancionadas permitió identificar el modo en que las instancias decisorias adoptaron las políticas públicas, mientras se avanzaba hacia un nuevo modelo educativo dentro de la Universidad, según los criterios orientadores del Espacio Europeo de Educación Superior.

Palabras clave: educación superior, universidad, política educativa, reforma.

\begin{abstract}
This paper presents an analysis of the Bologna Process understood from the Spanish University. The reformist implications of this process have driven a set of modifications that can be revised from a qualitative approach in order to determine their possible effects on the Spanish university education. Therefore, the interpretative analytical method was selected as a methodological line for developing this trial. Observation and analysis of primary and secondary sources are also considered. The observation made through different governments and regulations adopted made possible to identify how public policies were adopted by decision makers as they moved towards a new educational model in the University, in accordance with the guiding criteria by the European Higher Education Area.
\end{abstract}

Keywords: Higher Education, University, Educational Policy, Reform. 


\section{Introducción}

Una de las organizaciones humanas con más camino recorrido en la historia de la humanidad es la Universidad. Mientras avanza el desarrollo de los Estados y las naciones, se incrementa el grado de complejidad de dicha institución propiciando que se convierta en una institución dirigida principalmente a la transmisión de valores, el fomento del conocimiento y la socialización de la cultura. En consecuencia, "la Universidad moderna se caracteriza por su naturaleza contradictoria: tradición versus innovación, anquilosamiento versus renovación, estatismo versus dinamismo. Estos binomios hacen de la educación superior actual un ámbito en proceso evolutivo constante" (Sánchez y Zubillaga, 2005, p. 169). Sin embargo, es posible que la Universidad actual como institución sea el resultado de una suerte de combinación de varias formas institucionales y entre esas formas, el equilibrio se presente de forma inestable (Mires, 2014).

Así, una serie de cambios se han experimentado dentro de la educación universitaria española coincidiendo con el impulso del conocido Proceso de Bolonia. Dicho proceso implica, en este caso, que desde España se adopten los principios del Espacio Europeo de Educación Superior (en adelante, EEES) con lo cual, tanto los gobiernos como las Universidades han concebido líneas de acción que no siempre generan los resultados esperados, según los criterios orientadores del Proceso de Bolonia.

De forma paulatina, se adelantaron iniciativas nacionales e institucionales con el objeto de avanzar en la instauración de la nueva Universidad que reclamaba Bolonia.
Sin embargo, el tránsito hacia un nuevo ideario pedagógico, el contexto social y económico y las referencias regionales determinaron este proceso reformista, por lo cual, la Universidad española ha experimentado una serie de cambios bajo el subterfugio de la armonización con el EEES. Sin embargo, no todos los efectos fueron los esperados o quizás, no fueron los proyectados (Pereyra et al., 2006).

Este tema sobre la reforma universitaria a la luz de Bolonia ha provocado numerosas intervenciones desde múltiples puntos de vista: partidos políticos, académicos, estudiantes, investigadores y científicos han manifestado su opinión en torno a lo que ha supuesto para la Universidad española este nuevo rumbo universitario. Cada posición ideológica y cada convicción han tratado de interpretar cómo debe concebirse la institución universitaria. Esta afirmación permite inferir que no se trata de un cuerpo aislado o poco cercano; se trata de una institución socialmente pertinente a la que muchas veces se le exige más de lo que su capacidad le permite responder.

En este sentido y como contexto de esta investigación, se observan datos y cifras correspondientes a las proporciones en cuanto a las preferencias de estudios a cursar por parte de los estudiantes, antes y durante esta reforma. Por otro lado, el análisis profundo de la normativa española permite apreciar el grado de vinculación con los criterios orientadores del proceso de Bolonia. El objeto es ofrecer criterios de análisis que ayuden a construir no solo conocimiento que se relacione con la dinámica del sector universi- 
tario, sino que además pueda colaborar con la construcción de propuestas que sirvan de plataforma para proponer análisis cualitativos dentro del campo educativo.

\section{Metodología}

El epicentro que sirve de foco principal en este artículo es la revisión y análisis de la dinámica universitaria española a propósito de la puesta en marcha de lo que se conoce como Proceso de Bolonia. Esto se hace con el objeto de exponer un conjunto de elementos que fomenten el debate y la discusión de los hallazgos encontrados como resultado del ejercicio investigativo. Al identificar el objeto de investigación se pudo asumir como perspectiva metodológica la metodología cualitativa mediante la que el conocimiento se aprecia desde una dimensión cualitativo-fenomenológica. En este sentido, el saber que se obtiene como resultado de esta práctica investigativa se podrá emplear desde lo hipotético y desde lo contextual en función del entorno contemporáneo.

Lo anterior es compatible con el ejercicio analítico de las fuentes documentales (primarias y secundarias), ya que de esta manera el estudio analiza a profundidad un fenómeno de índole social. No obstante, la aproximación a estas fuentes facilita el reconocimiento de la situación actual del tema que aquí se profundiza, desde las propuestas teóricas de los autores consultados y sus respectivos enfoques. Por lo tanto, desde una perspectiva interpretativa y analítica se observa, infiere y analiza el tema objeto de la investigación desembocando en el ejercicio hermenéutico, mientras se acepta que las partes que componen el fenómeno pueden recibir interpretaciones diversas (Martínez, 2006, p. 135).

Se toma como espacio temporal el período comprendido entre 1992 y 2011 con el interés de identificar y revisar cómo se ha comportado el número de estudiantes universitarios. Además, se identificó el conjunto de políticas públicas españolas que se han enfocado de forma directa hacia el sector universitario, con el objeto de inferir cómo los diferentes gobiernos se han dirigido a este sector educativo (Partido Socialista Obrero Español, en adelante PSOE, y el Partido Popular, en adelante PP), con especial atención a la armonización normativa en relación con los criterios orientadores del EEES. A nivel global, las fases de esta investigación fueron las siguientes: Fase 1: reconocimiento del objeto de la investigación y revisión de las fuentes documentales. Fase 2: Recopilación y clasificación de los datos. Fase 3: Estudio y exploración de las políticas públicas gubernamentales y análisis de los datos y las cifras oficiales. Fase 4: Elaboración del informe de la investigación y sus conclusiones.

\section{Bolonia y la reforma universitaria}

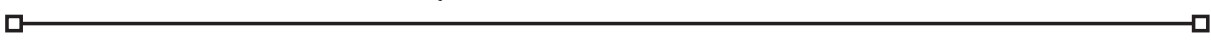

El Proceso de Bolonia se puede asumir como una suerte de detonante para que en España se produjera un conjunto de acciones que pretendieron, en su momento, armonizar la Universidad española en rela- ción con lo que se estaba generando en el contexto universitario europeo. Este interés amparado en la búsqueda de la adecuación del sistema universitario recibe el nombre de "armonización", ya que pretende unificar 
criterios tanto de forma como de fondo; sin embargo se reconoce que la llamada "Declaración de Bolonia”, no siendo una norma de derecho comunitario, no es vinculante (Sanjurjo, 2012). Por lo tanto, los países europeos se suscriben a los principios de esta declaración de forma voluntaria, aunque en la práctica pareciera que es imperativo el hecho de participar en este movimiento reformista precisamente para apoyar y fomentar, este caso, la integración de los estudios universitarios a nivel continental.

La práctica de esa armonización ha generado la aprobación de todo un entramado normativo no solo desde el gobierno central, también las propias universidades han impulsado una serie de acciones con la convicción de entender lo que se pretende con el Proceso de Bolonia, a partir del reconocimiento de un nuevo modelo educativo centrado en el estudiante y desde una autogestión de su propio conocimiento. Es de reconocer que, desde un punto de vista formal, los criterios orientadores del Proceso de Bolonia pueden considerarse deseables. No obstante, existen diferencias sustantivas entre la intencionalidad de tales criterios, la forma en la que estos son abordados y entendidos y evidentemente, lo que las universidades asumen como producto de lo que se infiere a partir de los principios rectores de dicho proceso.

Como era de esperarse, la llegada a España del Proceso de Bolonia generó todo un debate tanto a favor como en contra. Posiblemente, al momento de su llegada se necesitaba de una auténtica disposición política para que esa coyuntura en realidad sirviera como oportunidad para redefinir el quehacer universitario, de tal manera que los esfuerzos no se redujeran solo a la pro- ducción de normativas, sino que el seguimiento de la reforma universitaria estuviese acompañado de una profunda convicción de un cambio universitario con una perspectiva vinculante desde lo social. Según apuntan Ruiz-Gallardo y Castaño (2008), el avance de la reforma de la Universidad española podrá experimentar un camino positivo si se asumen básicamente dos iniciativas, 1.- La reducción del gran número de titulaciones para finalmente unificarlas al contexto europeo y 2.- Administrar el currículum a partir del sistema de transferencia de créditos europeos (ETCS).

Aplicando lo anterior, a nivel europeo se diseñó la estructura de las enseñanzas universitarias en tres ciclos: Grado $\left(1^{\circ} \mathrm{ci}-\right.$ clo), Máster ( $2^{\circ}$ ciclo) y Doctorado $\left(3^{\circ} \mathrm{ci}-\right.$ clo). En el contexto universitario español esta dinámica se asumió desapareciendo titulaciones como "Licenciaturas superiores" o "Diplomados". Martínez Ruiz y Sauleda Parés (2007) recuerdan que "un crédito equivale a un trabajo de entre 25 y 30 horas. Un año académico conlleva 60 créditos (1500-1800 horas)" (2007, p. 132). Por su parte, Ruiz-Gallardo y Castaño (2008) destacan la importancia de las horas en función de la labor que debe realizar el estudiante, ya que se asume como instrucción para la educación universitaria hacer énfasis en el aprendizaje. Esto significa que se reafirma que el centro de atención de la educación universitaria es el estudiante, con lo cual el docente universitario se concibe no como transmisor de conocimientos sino como acompañante y orientador del estudiante al momento en que este emprenda su tránsito por los estudios universitarios.

Se trata además de asumir que, ante esta perspectiva curricular, se debe redefinir 
el papel del docente universitario y siendo este de gran relevancia ha sido una ausencia notable dentro de toda la documentación que se ha generado en torno al Proceso de Bolonia. Así, se ha facilitado que desde las propias universidades se implementen planes de formación destinados a la mejora didáctica de la docencia universitaria, pero se espera una mayor vinculación por parte de los cuerpos decisorios a nivel central (Mas-Torelló y Olmos-Rueda, 2016).

La formación docente universitaria no ha estado siempre en el objetivo político de las grandes reformas impulsadas para la Universidad. En las reuniones de encargados del área universitaria europea sí se ha señalado el impulso a esta actividad, pero resulta necesario reconocer la situación real del profesorado universitario desde una profunda revisión de sus condiciones laborales y la distribución de los tiempos previstos para tal fin; además de la retribución económica de su labor. Lógicamente, el profesorado universitario que tiene la responsabilidad de adecuarse a las reformas que se establezcan también va a necesitar acompañamiento y formación institucional, ya que no es suficiente ser experto en un campo de estudio para ser docente universitario y, desde luego, para conocer la didáctica propia de este sector educativo (Girona et al., 2018). Sin embargo, y sin restar importancia a este aspecto, por lo general, los académicos se encuentran sumergidos en un sistema de méritos que muchas veces constituye una auténtica carrera de obstáculos y no siempre se suele reconocer el valor de la docencia en su justa dimensión.

Para todo docente, en este caso el docente universitario, siempre será un reto despertar en sus estudiantes la pasión por el conocimiento. No se trata de intentar "enseñar" una determinada técnica o un conjunto de principios teóricos, más bien se habla de que los docentes universitarios se conviertan en diseñadores de escenarios de aprendizaje (Salinas et al.,2014) en los que puedan orientar y acompañar a sus estudiantes en su tránsito por la formación universitaria, de forma tal, que en esos escenarios se pueda trabajar por ejemplo desde el análisis de un caso "en un contexto educativo del futuro, y el proceso de creación de estos escenarios ayuda a los implicados en la planificación del cambio a que tengan una mejor comprensión de todo el proceso" (Salinas, 2012, p. 8). Esta concepción dista mucho de llevarse a cabo si se continúa de forma tradicional y con un desbalance en cuanto a la dedicación que conlleva la carrera académica.

Ahora bien, este nuevo escenario que ya se acepta como un auténtico reto o como algo más que se agrega a la lista de deberes que se espera que cumplan los académicos, no deja de suponer lógicamente una necesidad formativa (Imbernón, 2012). Así, se destaca la necesidad de que las universidades tengan la capacidad para proponer un currículum que se acerque lo más posible a lo que un contexto determinado espera en cuanto al talento humano que egresa, que pueda atreverse inclusive a formar para profesiones que todavía no existen. Al explorar la dinámica experimentada respecto a los estudiantes y los estudios que estos han cursado, es importante analizar la información que maneja el Instituto Nacional de Estadística (INE) entre 1998 y 2010. Para tal fin, se expone a continuación la Figura 1 que expresa el porcentaje de los estudiantes según el área de estudio. 


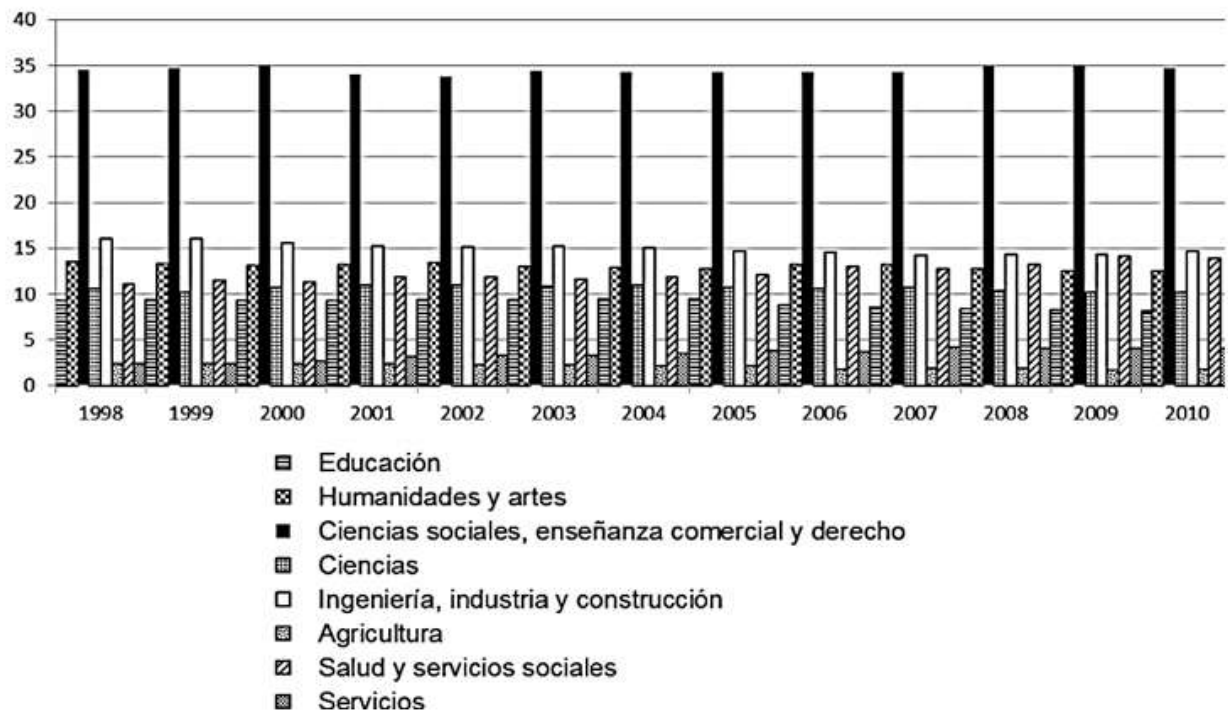

Figura 1: \% de todos los estudiantes por área de estudio (España)

Fuente: Elaboración del autor con datos tomados de:

EUROSTAT http://www.ine.es/jaxi/tabla.do?path=/t13/p405/e01/10/\&file=04002.px\&type=pcaxis\&L=0

Para analizar estos datos se puede hacer dos ejercicios, desde un nivel macro y un nivel micro. En primer lugar, a nivel macro se puede obtener una idea compatible de que en general no hay mayores variaciones, es decir, no se muestra un comportamiento significativo diferente a lo largo de los años observados. Pareciera más bien que el patrón se reproduce en todo el período. En segundo lugar, si se hace una revisión a nivel micro, se puede apreciar que el área de servicios tiende al alza y por el contrario el área de agricultura tiende a bajar. Todo esto ocurre en el año 2006 y, en ambos casos, se cuenta con pocos estudiantes matriculados. Por su parte, las áreas de servicios sociales, salud, ciencias, humanidades y artes, derecho, enseñanza comercial y las ciencias sociales tienen un número de estudiantes que se sostiene a lo largo de los años analizados y apenas tienen modificaciones. Este aspecto corresponde con la idea de que previa y posteriormente a la implementación del Proceso de Bolonia no se ha generado variación respecto al interés de los estudiantes al momento de cursar estudios universitarios, conservando el mayor número de estos en el área de derecho, enseñanza comercial y ciencias sociales. Si bien es cierto que entre 1998 y 2010 la dinámica social española ha experimentado modificaciones, esta no ha influido de forma determinante en cuanto a los intereses estudiantiles respecto a preferencias de índole universitaria. Mientras que en los países más industrializados el abandono de los estudios obedece mayoritariamente a nuevas oportunidades laborales, en el caso español, por ejemplo, hay un fuerte componente motivacional en los estudiantes que desertan (Cabrera, 2015).

Se puede inferir que la intencionalidad de la reforma universitaria en España, la cual se ha desarrollado a la luz del Proceso de Bolonia, no ha supuesto un volumen importante en cuanto a nuevas posibilidades para desarrollarse como profesionales 
ante los nuevos escenarios que se infiere están siendo demandados por la sociedad actual, ya que como se ha observado en las estimaciones anteriores, no hay un comportamiento significativamente diferente respecto a las preferencias por los estudios universitarios. Además, las iniciativas vinculadas con la adaptación y modernización curricular de los estudios universitarios quizás tiendan más hacia una titulación universitaria para responder a un requisito determinado, mientras que la competencia para aplicar lo aprendido en el mundo real generalmente se asuma desde la vinculación del estudiante con su futuro espacio de desarrollo laboral, no siempre de la mano de la Universidad como institución formadora. Así, y "hasta la fecha, la reforma de Bolonia no ha tenido mucho éxito por lo que se refiere al objetivo de reducir la inadecuación de las cualificaciones en la UE" (Parlamento Europeo, 2015, s.p.). Además, se hace necesario el fomento de perspectivas pedagógicas que permitan el ejercicio del pensamiento crítico, analítico e interpretativo en lugar de la realización de actividades más hacia lo memorístico.

El impacto del EEES ha sido influyente de forma notable si se ve desde tres esferas que se interrelacionan: la esfera supranacional (Políticas de la Unión Europea), la esfera nacional (los Estados signatarios interpretan los lineamientos europeos), y la esfera institucional (las universidades adoptan los lineamientos recibidos de las esferas anteriores) (Muga y Bruce, 2018). De tal manera, la dinámica reformista en Europa ha impulsado cambios de naturaleza estructural, que quieren estandarizar la manera de administrar la Universidad desde todas sus facetas, sin dejar de reconocer la particularidad de cada sistema universi- tario propio de los países (Escudero y Tri1lo, 2015).

En orden cronológico y hasta el presente, se han aprobado los siguientes documentos producidos en los encuentros de ministros europeos encargados del sector universitario de los países miembros: Magna Charta Universitatum (1988), Declaración de La Sorbona (1998), Declaración de Bolonia (1999), Comunicado de la reunión de ministros europeos responsables de la enseñanza superior, Praga (2001), Comunicado de la reunión de ministros europeos responsables de la enseñanza superior, Berlín (2003), Comunicado de la reunión de ministros europeos responsables de la enseñanza superior, Bergen (2005), Comunicado de Londres (2007), Comunicado de la reunión de ministros europeos responsables de la enseñanza superior, Lovaina (2009), Declaración de Budapest y Viena sobre el EEES (2010), el Comunicado de Bucarest (2012), la Declaración de Ereván (2015) y el Comunicado de París (2018).

Actualmente en el EEES, se considera el uso del ETCS como una manera de medir el grado de implantación de esta forma de administrar el currículum universitario en los países signatarios del Proceso de Bolonia. Así, el ETCS es el método concebido para transferir y acumular créditos que ya se asume en la mayoría de los países del EEES. Sin embargo, hay ocho países en los que se utiliza un sistema nacional de crédito para la acumulación y transferencia de créditos. Por ejemplo, en Belarús, Bulgaria, Kazajstán, Letonia, Suecia y el Reino Unido se exige el uso de un sistema nacional de crédito y se determinan las normas específicas de conversión entre ese sistema nacional y el ETCS. 
Por su parte, en la República Checa y el Reino Unido no hay requisitos formales para utilizar ningún sistema de créditos en la enseñanza superior. Sin embargo, en todos los países, incluidos los que tienen sistemas nacionales, el ETCS se utiliza en la práctica por todas o la mayoría de las instituciones de enseñanza superior al menos en el contexto de la movilidad internacional. En algunos países no se utiliza el ETCS para la acumulación dentro de las universidades ni para la transferencia de créditos entre instituciones a nivel nacional.

En un total de 45 sistemas de educación superior se observa que en las enseñanzas de primer y segundo ciclo se utiliza el ETCS, en comparación con 36 países en 2013 y 2014. Así, y desde el informe de 2015, se han registrado progresos en Kazajstán, Macedonia y Rusia. Son países en los que todos los programas utilizan actualmente el ETCS para la transferencia de créditos.

Hay algunas particularidades, por ejemplo, en Chipre, República Checa, Santa Sede e Irlanda ni todos los programas ni todas las instituciones utilizan el ETCS. En la comunidad flamenca de Bélgica, los programas pre-Bolonia se reorientan de forma gradual en términos de resultados de aprendizaje y los créditos del ETCS se asignan a los programas revisados. No obstante, para la Comisión Europea (2018) el uso del ETCS se usa prácticamente en toda Europa, con lo cual se puede asegurar que la administración curricular bajo los criterios orientadores del Proceso de Bolonia es una realidad de alcance continental.

Según las orientaciones acordadas en Europa, habitualmente la carga de trabajo de un estudiante está entre 1500 y 1800 horas por año o curso académico, con lo cual un crédito equivale entre 25 y 30 horas de trabajo. Por lo tanto, 60 créditos ETCS corresponden a un año académico a tiempo completo aplicable a todos los niveles que conforman el sistema de educación superior. Sin embargo, dentro de los países miembros del Proceso de Bolonia no existe un consenso generalizado particular al respecto. Por ejemplo, Bélgica (solo comunidad flamenca), Suiza, Chipre, República Checa, Grecia, España, Polonia, Eslovenia y Turquía tienen entre 1500 y 1800 horas por año académico y entre 25 y30 horas por crédito. Por otro lado, Bélgica (comunidad francesa) cuenta con 1440 horas por año académico y 24 horas por crédito. Otros casos observables son: Rumanía (1520 y 1640 horas por año académico y entre 25 y 27 horas por crédito), Austria, Italia y Malta (1500 horas por año académico y 25 horas por crédito), Dinamarca, Finlandia, Francia, Hungría y Países Bajos (entre 1600 y 1800 horas por año académico y entre 25, 27, 28 y 30 horas por crédito) (Comisión Europea, 2018).

A nivel europeo se ha acordado que las cualificaciones del primer ciclo normalmente incluyan entre 180 y 240 créditos ETCS; sin embargo, no existe uniformidad en cuanto a esta disposición. Así, existe una tendencia marcada a diseñar los grados con menos de 240 ETCS, es decir, entre 165 y 198 ETCS, con lo cual de 47 países miembros del Proceso de Bolonia, 28 de ellos han establecido que los grados tengan una duración alrededor de los tres años, es decir, el 59,57\% con respecto al total. Estas proporciones sugieren que los grados se desarrollan en tres años, pero no todos tienen la misma cantidad de créditos ETCS. 


\section{La normativa española universitaria y la reforma de Bolonia}

Un aspecto relevante al momento de analizar las iniciativas reformistas universitarias se refiere a las políticas públicas dirigidas a este sector educativo. Su importancia radica en el hecho de considerar que esas políticas públicas permitirán reconocer la intencionalidad o la forma en que se dirigen los cuerpos decisorios, en este caso, hacia el sector universitario, además de inferir el sentido que tiene para estos el impacto y la importancia de la Universidad para el desarrollo social. Se asume que la política pública es una línea de acción (Croce, 2017). Por lo tanto, no se trata solo de su formulación sino de su puesta en práctica y, en su momento, de la evaluación del desarrollo de esa línea de acción y su posible impacto. De esta forma "las políticas públicas son primordiales para promover o inhibir la transformación social, pues son decisiones gubernamentales con consenso de los demás sectores de la sociedad, destinadas al logro de objetivos razonables predeterminados" (Rodríguez, 2018, p. 17).

En el caso del análisis que aquí se expone, el cuerpo normativo que ha existido en España respecto al sector universitario se erige como oportunidad para revisar tanto la intencionalidad que acompaña la sanción de tales normativas, así como para reconocer el cambio que en su momento generó la aparición del Proceso de Bolonia, ya que para esto era necesaria la articulación de las normativas nacionales en correspondencia con los criterios orientadores establecidos para el EEES. De forma privilegiada, se espera que el efecto de la reforma universitaria pueda fomentar la vinculación efectiva entre el contexto universitario y el contexto laboral. Esto significa "un gran esfuerzo de coordinación entre las instituciones de edu- cación superior, las empresas, los agentes sociales y las Administraciones públicas" (Gutiérrez-Solana, 2016, p. 5).

En los gobiernos en los que se impulsaron las iniciativas normativas para armonizar el sistema universitario español al contexto europeo, dichas iniciativas fueron determinadas por las políticas públicas evidentemente dirigidas al sector universitario. Al revisar estas políticas se pude inferir la intencionalidad de los gobiernos respectivos y cómo estos entendían que debía desarrollarse y organizarse este sector educativo. $\mathrm{Al}$ asumir que se trata de un nuevo modelo educativo en el que se reafirma la atención principal en el estudiante, se acepta que los diseños curriculares deben organizarse desde las competencias esperadas con el fin de que los profesionales universitarios no solo mejoren su empleabilidad, sino que puedan asegurar la sinergia entre lo que aprenden en las aulas y su aplicabilidad en el mundo real (Sendín y Espinosa, 2014). Al intentar responder a esta finalidad se entendió que era necesaria la apertura hacia el sector privado de la economía para que las competencias profesionales respondan a lo que ese sector requiere.

Más adelante se expone una tabla comparativa (Tabla 1) que contiene los criterios orientadores del Proceso de Bolonia y cuáles de estos están presentes en los documentos normativos españoles que se han expuesto, en función de la armonización de la Universidad española al EEES. Para la construcción de este cuadro comparativo, se agruparon los criterios emanados del Proceso de Bolonia a partir de la revisión de las declaraciones de ministros y responsables europeos del área universitaria desde 1998 
hasta el año 2018. Una vez identificados, se procedió a diseñar la lista respectiva. Esta lista fue contrastada con la documentación normativa española que se ha producido en virtud del Proceso de Bolonia.

No obstante, es preciso acotar lo siguiente: 1.- La Ley orgánica 11/1983 de reforma universitaria sancionada en el gobierno del PSOE. Si bien se concibió antes de que naciera el Proceso de Bolonia, ya establecía la proyección de la Universidad española dentro de la integración europea. Por lo tanto, esta ley se analizó para efectos de esta comparación. 2.- Los documentos: "La integración del sistema universitario español en

Tabla 1: Presencia de los criterios orientadores del Proceso de Bolonia dentro de la normativa española

\section{Legislación española}

\begin{tabular}{|c|c|c|c|c|c|c|c|c|c|}
\hline Criterios orientadores del Proceso de Bolonia & 1 & 2 & 3 & 4 & 5 & 6 & 7 & 8 & 9 \\
\hline Impulso del ETCS & & $\mathrm{X}$ & $\mathrm{X}$ & $\mathrm{X}$ & $\mathrm{X}$ & $\mathrm{X}$ & $\mathrm{X}$ & & \\
\hline Promoción del aprendizaje de idiomas & & & & & & & & & $\mathrm{X}$ \\
\hline Educación superior centrada en el estudiante & & & $\mathrm{X}$ & & & $\mathrm{X}$ & & & $\mathrm{X}$ \\
\hline Máster y Doctorado con mayor duración & & & $\mathrm{X}$ & $\mathrm{X}$ & & & & & \\
\hline Movilidad estudiantil & & $X$ & & $\mathrm{X}$ & $X$ & $X$ & & & \\
\hline Movilidad profesoral & & $\mathrm{X}$ & & & & $\mathrm{X}$ & & & \\
\hline Promoción internacional del sistema universitario & & $X$ & $X$ & & $\mathrm{X}$ & $\mathrm{X}$ & & & \\
\hline Políticas públicas correspondientes al Proceso de Bolonia & & $\mathrm{X}$ & $\mathrm{X}$ & $\mathrm{X}$ & $X$ & $\mathrm{X}$ & & & \\
\hline $1^{\text {o }}$ ciclo con una duración de 3 años (mínimo) & & $\mathrm{X}$ & & $\mathrm{X}$ & & $\mathrm{X}$ & & & \\
\hline Promoción de la cooperación europea & & & $\mathrm{X}$ & $\mathrm{X}$ & $\mathrm{X}$ & $\mathrm{X}$ & & & \\
\hline Integración de la formación e investigación & & & $\mathrm{X}$ & $\mathrm{X}$ & $\mathrm{X}$ & & & & \\
\hline $\begin{array}{l}\text { Desarrollo de un sistema de titulaciones comparable/ } \\
\text { reconocible }\end{array}$ & & $\mathrm{X}$ & $\mathrm{X}$ & $\mathrm{X}$ & $\mathrm{X}$ & $\mathrm{X}$ & $\mathrm{X}$ & & \\
\hline Calidad y su aseguramiento desde la cooperación europea & & & $\mathrm{X}$ & $\mathrm{X}$ & $\mathrm{X}$ & & $\mathrm{X}$ & & \\
\hline Aprendizaje a lo largo de la vida & & & $\mathrm{X}$ & $\mathrm{X}$ & $X$ & $X$ & & & \\
\hline \multicolumn{10}{|l|}{ Desarrollo de una red de trabajo en el $3^{\circ}$ ciclo } \\
\hline $3^{0}$ ciclo con formación interdisciplinar & & & $\mathrm{X}$ & & & & & & \\
\hline
\end{tabular}




\section{Legislación española}

\begin{tabular}{|c|c|c|c|c|c|c|c|c|}
\hline Revisión de la normativa propia de los estudios doctorales & $\mathrm{X}$ & $\mathrm{X}$ & $\mathrm{X}$ & & $\mathrm{X}$ & $\mathrm{X}$ & & \\
\hline $\begin{array}{l}\text { Iniciativas para favorecer la financiación del proceso } \\
\text { EEES }\end{array}$ & & $\mathrm{X}$ & $\mathrm{X}$ & & & & & \\
\hline Planes nacionales para mejorar la calidad & & & $X$ & $X$ & $\mathrm{X}$ & $X$ & $\mathrm{X}$ & \\
\hline $\begin{array}{l}\text { Alianzas estratégicas entre educación investigación y } \\
\text { empresa }\end{array}$ & & $\mathrm{X}$ & $\mathrm{X}$ & & $\mathrm{X}$ & $\mathrm{X}$ & & $\mathrm{X}$ \\
\hline Asignación económica necesaria para las universidades & $\mathrm{X}$ & $\mathrm{X}$ & $\mathrm{X}$ & & & & & $\mathrm{X}$ \\
\hline Universidad, financiación pública y desarrollo & & & $X$ & $X$ & & & & \\
\hline $\begin{array}{l}\text { Gobernanza del sector universitario y diálogo sobre } \\
\text { financiación }\end{array}$ & & $\mathrm{X}$ & & & $\mathrm{X}$ & $\mathrm{X}$ & & \\
\hline Enseñanzas universitarias de tres ciclos & & & & $X$ & $\mathrm{X}$ & & & \\
\hline $\begin{array}{l}\text { Flexibilidad en planes de estudio/programas } \\
\text { interuniversitarios }\end{array}$ & & & & & $\mathrm{X}$ & $\mathrm{X}$ & & \\
\hline Estudios universitarios desde la pertinencia social & & $\mathrm{X}$ & & & & $\mathrm{X}$ & & \\
\hline Mejora de la empleabilidad de los titulados & & & $\mathrm{X}$ & & & $X$ & & $\mathrm{X}$ \\
\hline Reconocimiento de la autonomía académica y universitaria & $\mathrm{X}$ & $\mathrm{X}$ & $\mathrm{X}$ & $\mathrm{X}$ & $\mathrm{X}$ & $\mathrm{X}$ & & \\
\hline \multicolumn{9}{|l|}{ Medio ambiente y el fomento de su aprendizaje } \\
\hline Fomento de la innovación en la enseñanza & & & & $\mathrm{X}$ & & & & \\
\hline Universidades con estructuras gerenciales eficientes & $\mathrm{X}$ & & $\mathrm{X}$ & & $\mathrm{X}$ & $\mathrm{X}$ & & \\
\hline \multicolumn{9}{|l|}{ Reconocimiento del aprendizaje no formal e informal } \\
\hline La Universidad como servicio público & $X$ & $X$ & & & & & & \\
\hline Evitar la exclusión universitaria por motivos económicos & & $\mathrm{X}$ & & & & $\mathrm{X}$ & & \\
\hline
\end{tabular}

Fuente: Elaboración del autor a partir de los datos revisados en la normativa española (2018).

La lista de documentos de análisis fueron los siguientes:

1) Ley orgánica $11 / 1983$, de reforma universitaria.

2) Ley Orgánica 6/2001, de Universidades.

3) Ministerio de Educación, Cultura y Deporte -MECD- (2003). La integración del sistema universitario español en el
Espacio Europeo de Enseñanza Superior, Documento Marco.

4) Ministerio de Educación, Cultura y Deporte - MECD - (2006). Documento de trabajo sobre La organización de las enseñanzas universitarias en España.

5) Ley Orgánica 4/2007, por la que se modifica la Ley Orgánica 6/2001, de Universidades. 
6) Real Decreto 1393/2007, de 29 de octubre, por el que se establece la ordenación de las enseñanzas universitarias oficiales.

7) Real Decreto 861/2010, que modifica el Real Decreto 1393/2007, por el que se establece la ordenación de las enseñanzas universitarias oficiales.

8) Real Decreto - Ley 14/2012, de medidas urgentes de racionalización del gasto público en el ámbito educativo.

9) Ley Orgánica 8/2013, para la mejora de la calidad educativa.

Se afirma que existe coincidencia en los documentos analizados en términos de los criterios que el Proceso de Bolonia establece para los países signatarios. En este caso, España mostró adopción de líneas de acción relacionadas con: impulsar el ETCS y Políticas públicas compatibles con el espíritu del Proceso de Bolonia, asumir un sistema de titulaciones reconocible y comparable, reforzar la reglamentación sobre el tercer ciclo, planes nacionales para mejorar la calidad, alianzas estratégicas entre la educación la investigación y las empresas, reconocimiento de la autonomía académica y universitaria. Es decir, los aspectos referidos fueron considerados por todos los documentos (leyes, reales decretos, documentos de trabajo y documentos marco). Sin embargo, la adecuación del sistema universitario español al EEES implica la modificación de metodologías de aprendizaje más allá del apoyo de un sistema que contabilice el trabajo del estudiante. En consecuencia, los documentos muestran una tendencia hacia el mejoramiento del proceso formativo que fomente el crecimiento social y económico.

Existen criterios que no son comunes en todos los documentos analizados. Es de destacar que deben considerarse de forma obligatoria al igual que los criterios señalados en el párrafo anterior, ya que constituyen el engranaje que posibilita el desarrollo efectivo del Proceso de Bolonia, según las metas a nivel europeo. Un aspecto a observar es que existe ausencia en la promoción del aprendizaje de idiomas, oportunidad para el impulso de la movilidad tanto estudiantil como profesoral. Por otra parte, no se evidencian menciones directas a la creación de redes de trabajo en el $3^{\circ}$ ciclo, ni el carácter interdisciplinar en estos estudios. Es posible que esto se deba a que la legislación española reconoce la autonomía académica de las universidades para la construcción de sus propias propuestas formativas; por lo tanto, se infiere que este aspecto le compete a las Universidades. En este sentido, la relevancia dada a la investigación desde Bolonia ofrece un panorama integrador entre la naturaleza propia de la Universidad y la finalidad académica de los estudios doctorales. En otras palabras:

Las críticas al racionalismo y al cientificismo más duro nos han hecho tomar consciencia de la necesidad de integrar campos, enfoques culturales, cosmovisiones, intersectorialidades, transversalidades. [...] En este marco, los sistemas educativos sufren una impresionante presión social que les exige respuestas a una demanda que los superan por todos los flancos. (Croce, 2017, p. 191)

El aspecto de la financiación hacia el sector universitario no es un punto en común dentro de los documentos analizados. Tal vez se pueda decir que, de manera tími$\mathrm{da}$, este tema ha sido apenas mencionado en proporción inversa a su relevancia. Se destaca además el Real Decreto, Ley 14/2012 de 20 de abril, de medidas urgentes de ra- 
cionalización del gasto público en el ámbito educativo sancionado en el gobierno del PP. En él se establecen de manera expresa los criterios para el diseño y ejecución del gasto universitario, en correspondencia con las palabras expresadas en la toma de posesión del gobierno en las que se destacó la posibilidad del ajuste y reducción de todas las partidas presupuestarias. Esto se traduce en que los recortes "han afectado sobre todo al personal de las universidades y centros de investigación, así como a las partidas de inversiones e infraestructuras" (Conesa y González, 2018, p. 258).

Continuando con este análisis documental, la Universidad como servicio público no figura como elemento de reconocimiento común dentro de los documentos revisados. Efectivamente solo la Ley orgánica 11/1983, de reforma universitaria, sancionada en el gobierno del PSOE y la Ley Orgánica 6/2001, de 21 de diciembre, de universidades sancionadas en el gobierno del PP hacen mención directa a este aspecto. El resto de los documentos refieren el rol de la Universidad como eje de desarrollo social y económico además de su papel en la investigación. Así mismo, el Proceso de Bolonia señala el reconocimiento del aprendizaje no formal e informal dentro de la vida universitaria; sin embargo ninguno de los documentos revisados lo menciona. No obstante, y durante el gobierno del PSOE se sancionó el Real Decreto 1892/2008, de 14 de noviembre, por el que se regulan las condiciones para el acceso a las enseñanzas universitarias oficiales de grado y los procedimientos de admisión a las universidades públicas españolas. En el Artículo 27 se estableció que:

Podrán acceder a la Universidad española en las condiciones determinadas en los artículos siguientes las personas que hubieran cumplido 25 años de edad, las que hubieran cumplido 40 años y acrediten una determinada experiencia profesional o laboral, así como las personas que hubieran cumplido 45 años. (Real Decreto 1892/2008).

Esta es la referencia al punto antes señalado. De tal manera se establecieron los criterios normativos destinados al reconocimiento del aprendizaje no formal e informal. En el año 2006, en España se expuso el documento: "La organización de las enseñanzas universitarias en España", presentado por el entonces Ministerio de Educación y Ciencia (PSOE), en el cual se establecía un cronograma que incluía las actividades a desarrollar por parte del ejecutivo nacional, en virtud de la armonización de la Universidad española al EEES. Tal y como se identifica en dicho documento, se acordó aprobar las siguientes normativas: a.- Enseñanzas de Grado y Postgrado, b.- Directrices para la elaboración de títulos, c.- Directrices propias de títulos de profesiones reguladas, d.- Registro de universidades, centros y titulaciones, e.- Homologación de títulos, f.- Estatuto del PDI, g.- Conferencia General de Política Universitaria, h.- Consejo de Universidades, i.- Creación de Centros y Universidades.

Por otro lado, se proyectó la oferta de matriculaciones para las nuevas titulaciones para mayo de 2008, de manera que para el mes de julio 2012 se tenía previsto el egreso de los nuevos titulados universitarios de grado bajo la reforma de Bolonia. Sin embargo, no se manifestó de manera expresa la normativa necesaria para el establecimiento de una planificación presupuestaria conforme a los requerimientos propios del proceso reformista universitario, ya que posiblemente este punto sería tratado en el seno de la Conferencia General de Política Universitaria o en el Consejo de Universidades. De igual 
modo, el plan de formación al profesor universitario no se señaló bajo una normativa particular. Se cuenta para esto con las inicia- tivas de cada Universidad desde su autonomía académica y sus particularidades como institución.

\section{Conclusiones}

Por lo general, cuando los cambios son impuestos o sugeridos desde fuera producen resultados diferentes a los esperados y en el caso de la Universidad no resulta diferente. A lo largo del desarrollo del Proceso de Bolonia se ha fomentado una serie de criterios orientadores que intentan armonizar los sistemas universitarios con el fin de que los procedimientos, la administración curricular y las competencias puedan tener un hilo común que los relacione. Esta idea ha tenido muchas aristas para revisar, ya que se ha decidido prácticamente de forma unilateral lo que la Universidad debe ser y hacer sin tener en cuenta el aporte o la opinión que las propias universidades puedan exponer respecto a lo que se decide sobre ellas.

De este modo, el desarrollo del Proceso de Bolonia ha sido asincrónico en toda Europa y en el caso español no ha sido diferente. Es de reconocer que las universidades españolas inician sus propios procesos internos de adaptación una vez que conocen el movimiento reformista europeo $\mathrm{y}$, posteriormente, el Estado español expone una serie de normativas y decretos con el objeto de unificar criterios de funcionamiento. Además de esta particularidad, se ha observado que el Proceso de Bolonia tiene sus principios y tanto las universidades como los estados han comprendido de forma muy diferente lo que hay que hacer. Mientras esto se desarrollaba, los estudiantes y los académicos debían asumir una postura pragmática entre la racionalidad y la incertidumbre ante lo que de momento no estaba del todo claro.
Con respecto a los instrumentos normativos generados desde España, se reconoce que la generación de normativas fue abrupta y que la aparición de una tras otra trajo como consecuencia una inestabilidad institucional considerable, ya que en muchos casos no se había terminado de asumir una instrucción cuando ya aparecía una normativa diferente. Por otra parte, si bien se ha observado la correspondencia entre los principios orientadores del Proceso de Bolonia y el marco normativo español, esta correspondencia obedece más a lo formal que a lo técnico-operativo. Es decir, en los textos se observa la adecuación de las normativas españolas al proceso de Bolonia; sin embargo, no implica que en la práctica se produzcan los cambios que se esperan de una reforma de esta magnitud.

Por otra parte, y como se ha revisado en las proporciones matriculares, no se ha generado una variación significativa respecto a las preferencias al momento de decantarse por estudiar titulaciones en un campo determinado del conocimiento. Por lo tanto, antes y después de Bolonia los estudiantes muestran los mismos intereses con lo cual se puede inferir que, en este caso, los cambios que se esperaban conseguir con esta reforma universitaria no guardaban estrecha relación con el futuro contexto social que recibiría el talento humano formado en las universidades. Además, las numerosas manifestaciones de protestas estudiantiles en contra del Proceso de Bolonia han traído como consecuencia una gran desafección al 
sistema universitario español, ya que se ha popularizado la idea de que la Universidad se ha convertido en un espacio privatizador del conocimiento, con poca vinculación social y una notable influencia de parte del sector privado de la economía en cuanto a la formación de personas según sus intereses económicos.

Las iniciativas reformistas dirigidas a la Universidad española han encontrado un conjunto de retos a superar básicamente por el hecho de que en la práctica se tiende a reproducir la misma dinámica docente. Es decir, desde Bolonia se espera que el centro de atención sea el estudiante y esto significa que este debe administrar, hacerse dueño y comprometerse con su aprendizaje, su descubrimiento y todo lo que esto implica, además de crecer en cuanto al pensamiento crítico, analítico y capacidad argumental; mientras tanto, el docente universitario asume la postura de orientador, acompañante y facilitador de este proceso. No obstante, en la práctica pareciera que se sigue reproduciendo el mismo esquema docente anterior a Bolonia, donde el docente era el dueño del saber y los estudiantes debían intentar a toda costa apropiarse y hasta reproducir ese saber. Aunque la presencia en la educación universitaria de las TIC sea una realidad, existe cierto escepticismo a propósito de la realidad que aquí se menciona.

Así las cosas, resulta difícil emprender un cambio profundo en la Universidad si no se está convencido de la necesidad de cambiar para mejorar. Con la reforma universitaria sí que es cierto que se ha avanzado en cuanto a la sistematización de un conjunto importante de procedimientos administrativos estandarizados a nivel europeo, solo que la administración curricular no puede ni debe estar divorciada del diseño curricular. Mucho menos si se sigue con la balcaniza- ción curricular en las facultades y departamentos.

Pareciera que la crisis institucional no solo es una característica de la Universidad, sino que además posiblemente sea su modo de existir. El repensar y redefinir el sentido de la institución universitaria conforme avanzan las sociedades es el papel que de forma permanente acompaña la cotidianidad de la Universidad. Mientras esto ocurre, resulta complicado lograr un balance entre el estilo de Universidad medieval, la Universidad como aparato estatal y la sinergia entre Universidad y empresa.

A propósito, esta dificultad radica en el hecho de que en torno a la institución universitaria son muchos los intereses que le rodean. Esos intereses básicamente tienen que ver con una cuota de poder determinada no solo en el seno de la Universidad, sino por aspectos externos como el sistema político y la filiación ideológica que también tienen su participación dentro del quehacer universitario. Al estar presente esta particularidad, la Universidad se balancea entre su propia naturaleza para seguir existiendo y las posibles necesidades económicas y de mercado que deben ser atendidas desde las universidades.

Otro aspecto no menos importante es el papel que se ha asumido respecto a la formación del docente universitario. Es importante dejar claro que ser especialista en una disciplina o campo de estudio, o ser un investigador, no es garantía automática de formación en didáctica para la docencia universitaria. Esto se complica cuando se pretende alejar esa práctica didáctica de tradiciones anteriores para dar paso a nuevas propuestas innovadoras cónsonas con lo que se espera de la reforma universitaria. Al parecer, resulta necesario que se asuma como política 
de Estado la formación didáctica del docente universitario y el acompañamiento que se pueda ofrecer a las universidades para garantizar esta formación.

En fin, la Universidad siempre será un blanco de iniciativas reformistas y siempre tendrá muchos retos a superar. La contradicción natural que habita en las instituciones universitarias se erige como centro de interés para que la universalidad del pensamiento, la pluralidad, el debate y la transmisión de valores y cultura tenga su espacio dentro de la definición de Universidad.

\section{REFERENCIAS BIBLIOGRÁFICAS}

Cabrera, L. (2015). Efectos del proceso de Bolonia en la reducción del abandono de estudios universitarios: datos para la reflexión y propuestas de mejora. Revista Fuentes, 16, 39-62. Recuperado de http://www.revistafuentes.es/

Conesa, E., y González, A. (2018). Neo-gerencialismo y austeridad en el contexto académico español y europeo. ¿Dos caras de la misma moneda? Política y Sociedad, 55(1), 257 - 282.

Croce, A. (2017). Políticas públicas de educación: Estado y ONG’s. Colección, (11), 187-197.

Escudero, J., y Trillo, F. (2015). Un análisis crítico del Espacio Europeo de Educación Superior como reforma de la enseñanza universitaria: los programas Verifica y Docentia en el contexto español. Educar em Revista, 31(57), 81-97.

European Commision/EACEA/Eurydice (2018). The European Higher Education Area in 2018: Bologna Process Implementation Report. Luxembourg: Publications Office of the European Union.

Girona, C., Guàrdia, L., y Mas, X. (2018). La docencia universitaria más allá del 2020: tendencias, retos y nuevos escenarios. En: S. Carrasco e I. De Corral (Eds.) Docencia universitaria e innovación (pp. 195-226). Barcelona: Octaedro.

Gutiérrez-Solana, F. (2016). Políticas universitarias para una nueva década: problemas y oportunidades. La cuestión universitaria, (6), 4-11.

Imbernón, F. (2012). La formación del profesorado universitario: orientaciones y desorientaciones. Las prácticas de formación del profesorado universitario. En J. Bautista (coord.) Innovación en la universidad. Prácticas, políticas y retóricas (pp. 85-102). Barcelona: Graó.

Ley orgánica 11/1983, de 25 de agosto, de reforma universitaria. BOE-A-1983-23432

Ley Orgánica 2/2006, de 3 de mayo, de Educación. BOE-A-2006-7899

Ley Orgánica 4/2007, de 12 de abril, por la que se modifica la Ley Orgánica 6/2001, de 21 de diciembre, de Universidades. BOE-A-2007-7786. 
Ley Orgánica 6/2001, de 21 de diciembre, de Universidades. BOE-A-2001-24515.

Ley Orgánica 8/2013, de 9 de diciembre, para la mejora de la calidad educativa. BOE-A-2013-12886.

Martínez Ruiz, M. Á., y Sauleda Parés, N. (2007). Glosario EEES: terminología relativa al Espacio Europeo de Educación Superior. Universidad de Alicante.

Martínez, P. (2006). El método de estudio de caso: estrategia metodológica de la investigación científica. Pensamiento \& gestión, (20), 165-193.

Mas-Torelló, Ó., y Olmos-Rueda, P. (2016). El profesor universitario en el Espacio Europeo de Educación Superior: la autopercepción de sus competencias docentes actuales y orientaciones para su formación pedagógica. Revista mexicana de investigación educativa, 21(69), 437-470.

Ministerio de Educación y Ciencia (2006) La organización de las enseñanzas universitarias en España. Documento de trabajo. Recuperado de http://www.aedean.org/ANECA/PropuestaMECSep06.pdf

Ministerio de Educación, Cultura y Deporte [MECD]. Secretaría General de Universidades. Subdirección General de Coordinación y Seguimiento Universitario. (2013). Datos básicos del sistema universitario español. Curso 2013-2014. Secretaría General Técnica. Subdirección General de Documentos y Publicaciones.

Mires, F. (2014). La crisis de la Universidad. Paradigma: Revista de investigación educativa, 20(31), 9-18.

Muga, A., y Bruce, G. (2018). La declaración de Bolonia y su impacto para las políticas públicas en educación superior: internacionalización y competitividad en un mundo globalizante. Calidad en la Educación, (22), 161-173.

Parlamento Europeo. Resolución del Parlamento Europeo, de 28 de abril de 2015, sobre el seguimiento de la aplicación del Proceso de Bolonia. http://eur-lex.europa.eu/legal-content/ES/TX$\mathrm{T} /$ ?uri=CELEX\%3A52015IP0107

Pereyra, M., Luzón, A. y Sevilla, D. (2006). Las universidades españolas y el proceso de la construcción del Espacio Europeo de Educación Superior. Limitaciones y perspectivas de cambio. Revista Española de Educación Comparada. 12, 113-144.

Real decreto - Ley 14/2012, de 20 de abril, de medidas urgentes de racionalización del gasto público en el ámbito educativo. BOE-A-2012-5337.

Real Decreto 1393/2007, de 29 de octubre, por el que se establece la ordenación de las enseñanzas universitarias oficiales. BOE-A-2007-18770

Real Decreto 1892/2008, de 14 de noviembre, por el que se regulan las condiciones para el acceso a las enseñanzas universitarias oficiales de grado y los procedimientos de admisión a las universidades públicas españolas. BOE-A-2008-18947. 
Real Decreto 861/2010, de 2 de julio, por el que se modifica el Real Decreto 1393/2007, de 29 de octubre, por el que se establece la ordenación de las enseñanzas universitarias oficiales. BOE-A-2010-10542.

Rodríguez, J. (2018). Políticas públicas. Revista Venezolana de Enfermería, 4(2), 17-26.

Ruiz-Gallardo, J. R., y Castaño, S. (2008). La Universidad española ante el reto del EEES. Docencia e Investigación: Revista de la Escuela Universitaria de Magisterio de Toledo, 18, 253-270.

Salinas, J. (2012). La investigación ante los desafíos de los escenarios de aprendizaje futuros. Revista de educación a distancia, (50), 1-24.

Salinas, J., De Benito, B., y Lizana. A. (2014). Competencias docentes para los nuevos escenarios de aprendizaje. Revista interuniversitaria de formación del profesorado, (79), 145-163.

Sánchez, P, y Zubillaga, A. (2005). Las universidades españolas ante el proceso de convergencia europeo: análisis de las medidas institucionales y acciones de aplicación y coordinación. Revista de educación, (337), 169-187.

Sanjurjo, V. (2012). El proceso de Bolonia: mito y realidad. Revista Jurídica de Investigación e Innovación Educativa. (5), 121-146.

Sendín, M., y Espinosa, M. (2014). La Universidad tras el proceso de Bolonia: ¿una Universidad reformada o una Universidad necesitada de reforma? AFDUC, (18), 525-548. 